\title{
How Males Can Gain by Harming Their Mates: Sexual Conflict, Seminal Toxins, and the Cost of Mating
}

\author{
Rufus A. Johnstone ${ }^{1, *}$ and Laurent Keller ${ }^{2, \dagger}$
}

1. Department of Zoology, University of Cambridge, Downing Street, Cambridge CB2 3EJ, United Kingdom;

2. Institute of Zoology and Animal Ecology, Bâtiment de Biologie, University of Lausanne, CH-1015 Lausanne, Switzerland

Submitted February 12, 1999; Accepted May 26, 2000

ABSTRACT: We suggest that damaging mating tactics, such as physical aggression, the evolution of genital barbs and spines, and the transfer of seminal toxins may serve as a general means by which males can induce females to avoid or to delay remating. Provided that cumulative damage has an accelerating impact on fitness, a female who has already been harmed by previous partner(s) may do best to refrain from remating to avoid suffering still further damage. Consequently, a male can gain through the imposition of mating costs, even though this may reduce female fitness because by doing so he minimizes the chances that his mate will copulate again. We develop a game theoretical model of this possibility, focusing on toxin transfer as an illustrative example. We show that toxicity as a means of inhibiting remating is phenotypically stable over a broad range of conditions (although, under some circumstances, it may be necessary to invoke other selective pressures to account for the initial evolution of toxicity). The model predicts that toxin transfer should be more common (and involve greater levels of toxicity) in species with greater last-male mating advantage; it is also most likely where the poison inflicts strongly accelerating, dose-dependent costs on females.

Keywords: sexual conflict, mating systems, manipulation, arms race.

Females of many animal species often mate with more than one partner. This may yield a range of possible benefits, including replenishment of sperm, fertility insurance, seminal feeding, and (more controversially) increased genetic diversity of offspring (Walker 1980; Thornhill and Alcock 1983; Ridley 1988, 1993; Madsen et al. 1992; Lewis and Austad 1994; Keller and Reeve 1995; Yasui 1998; Jennions and Petrie 2000). From the perspective of an in-

*E-mail: raj1003@hermes.cam.ac.uk.

† E-mail: laurent.keller@ie-zea.unil.ch.

Am. Nat. 2000. Vol. 156, pp. 368-377. (c) 2000 by The University of Chicago. 0003-0147/2000/15604-0004\$03.00. All rights reserved. dividual male, however, subsequent remating by a female with whom he has copulated is almost inevitably disadvantageous, since it leads to sperm competition and possible loss of paternity. Consequently, there is a potential conflict of interest between the sexes over female mating behavior, and selection will favor males that are able to reduce the chances of or delay subsequent copulation by their mates (Parker 1970, 1984; Simmons and Siva-Jothy 1998).

Males can prevent females from remating by fending off rivals (see, e.g., Birkhead 1998; Gomendio et al. 1998; Olsson and Madsen 1998), by sealing the female reproductive tract with a mating plug (see, e.g., Baur 1998; Simmons and Siva-Jothy 1998), or by interfering with the process of mate attraction (e.g., Happ et al. 1970). Alternatively (or additionally), they can manipulate female behavior to reduce the propensity for remating. In many insect species, for example, males are known to transfer within the ejaculate substances that render females sexually unreceptive and that promote oviposition (Thornhill and Alcock 1983; Chen 1984; Cordero 1995; Simmons and Siva-Jothy 1998).

Here, we suggest that males may also influence female reproductive decisions through the imposition of mating costs. A number of studies have shown that copulation can have damaging consequences for females. For instance, in the fruit fly Drosophila melanogaster, male accessory gland products transferred to females during mating are toxic, causing females with elevated rates of mating to die younger than controls (Fowler and Partridge 1989; Chapman 1992; Chapman et al. 1995; Rice 1996). Furthermore, there is evidence suggesting similar mating costs in other species of Diptera (Chapman et al. 1998) and in the mite Caloglyphus berlesei (Radwan and Rysinska 1999). Physical damage to the female reproductive tract as a result of mating has been reported in the bushcricket Metaplastes ornatus (von Helversen and von Helversen 1991) and in the bruchid beetle Callosubruchus maculata (Simmons and Siva-Jothy 1998; see also Eberhard 1985), and injury as a result of aggressive male mating tactics is common in some mammals and birds (Daly 1978; LeBoeuf and Mesnick 
$1991 a, 1991 b)$. We propose that selection may favor males who inflict such costs on their partners because by doing so, they discourage remating.

Our argument can be summed up as follows, using the transfer of seminal toxins as an illustrative example: provided that the acquisition of greater quantities of toxin has an increasingly deleterious effect on fitness (see Chapman et al. 1995 for evidence of a dose-dependent effect), a female who has received a large amount from her partner(s) may do best to refrain from remating to avoid receiving still more of the harmful substance. Consequently, an individual male can gain by transferring a large dose of the toxin, even though this may reduce female fitness (and possibly his own reproductive output) because, by doing so, he inhibits remating and, thus, increases his expected share of paternity. Other harmful mating tactics may have an equivalent effect: if, for instance, accumulated physical damage from mating has an accelerating impact on fitness, an individual male can once again profit by inflicting harm on his mate because this will reduce her propensity for remating. Seminal toxicity, or the imposition of mating costs by any other means, may, thus, serve to manipulate female behavior.

Below, we present a game-theoretical model that examines this possibility. We examine the stability of seminal toxin transfer in a phenotypic, game-theoretical model and investigate the ease with which it may become established in a population, in relation to various parameters (the nature of the costs imposed by the toxin, the benefits that females stand to gain from remating, and the pattern of sperm competition). Although we focus on toxin transfer, we emphasize that the model is applicable to any form of cumulative damage inflicted during mating.

\section{The Model}

For simplicity, we will assume that females may mate either once or twice. In the absence of seminal transfer of toxins, the latter choice typically yields a net fitness benefit, which could reflect replenishment of sperm, fertility insurance, seminal feeding, or any combination of these factors (Walker 1980; Thornhill and Alcock 1983; Ridley 1988, 1993; Madsen et al. 1992; Lewis and Austad 1994; Keller and Reeve 1995; Yasui 1998). However, the benefit to be gained by remating varies from one female to another because of differences in nutritional condition, quantity of stored sperm, or ability to bear the costs of locating a new partner (see, e.g., Gromko et al. 1984; Harshman et al. 1988; Chapman et al. 1994 for evidence that such factors can influence propensity to mate). The fitness benefit of remating (relative to the mean fitness of a singly mated individual) will be denoted $f$, its probability distribution among females (which we assume to be continuously dif- ferentiable) $p(f)$, and the corresponding cumulative probability distribution $P(f)$. (Note that, for some individuals, $f$ may be negative, indicating that the costs of remating outweigh the benefits to be gained.)

Males may transfer variable quantities of toxin to their partner(s) during mating, which reduce female reproductive success. A female who acquires a total quantity $t$ of toxin from her partner or partners incurs an additive fitness cost (relative to the mean fitness of a singly mated individual) of $c(t)$. We assume that $c(0)=0$, that $c(t)$ is twice continuously differentiable, and that $c^{\prime}(t)$ and $c^{\prime \prime}(t)$ are both positive, that is, that the cost of the toxin increases with the amount acquired and does so at an accelerating rate. Accelerating costs have been recorded for a number of toxic substances (see, e.g., Bryan and Shimkin 1943; Murphy and Cheever 1968) and may, in some cases, reflect the action of homeostatic mechanisms that can successfully cope with small amounts of toxin but are overwhelmed by larger quantities (Shuey et al. 1994; Roger and Kavlock 1996). Successive doses are assumed to increase the total cost suffered, either through cumulative damage or because persistence of the toxin leads to a buildup in the maximum level to which the female is exposed.

Given that increasing quantities of toxin have an accelerating impact on reproductive success, a female's decision whether to remate or not should depend on the quantity of toxin transferred by her first mate, as well as the amount she is likely to acquire by remating. If she receives a large quantity from her first mate, she may do best to forgo the benefits of remating to avoid the adverse effects of acquiring still more of the harmful substance. (Note that the assumption of accelerating costs is essential to this argument.)

Formally, a female who receives a quantity $t$ of toxin from her first mate and can expect to receive $\hat{t}$ from her second should remate if, and only if,

$$
\begin{aligned}
& 1+f-c(t+\hat{t})>1-c(t) \\
& \Rightarrow f>c(t+\hat{t})-c(t),
\end{aligned}
$$

that is, if, and only if, the benefits she stands to gain from remating exceed some critical threshold. Provided that $c^{\prime \prime}(t)>0$ (i.e., that costs are accelerating), the greater the value of $t$ (i.e., the more toxin she acquires from her first mate), the higher this threshold becomes (i.e., the larger the benefit needed to make remating worthwhile).

Males may, thus, benefit by transferring large amounts of toxin, even though this adversely affects the reproductive success of their partners because by doing so, they can reduce the chance of a female remating and, thus, increase their expected share of paternity (we assume that the costs of toxin production are negligible compared to 
the reduction in female fitness; see "Discussion"). The expected fitness of an individual mutant male who transfers a quantity of toxin $t$ to his mates, given that other males in the population transfer a quantity $\hat{t}$ (and that females, who are assumed to be able to detect and respond to the amount of toxin they receive from an individual male, follow the optimal policy summarized above), will be denoted $w(t, \hat{t})$, and is given by

$$
\begin{aligned}
w(t, \hat{t})=\alpha\{ & \int_{-\infty}^{c(t+\hat{t})-c(t)} p(f)[1-c(t)] \mathrm{d} f \\
& +\int_{c(t+\hat{t})-c(t)}^{\infty} p(f)(1-m)[1+f-c(t+\hat{t})] \mathrm{d} f \\
& \left.+\int_{c(2 \hat{t})-c(\hat{t})}^{\infty} p(f) m[1+f-c(\hat{t}+t)] \mathrm{d} f\right\},
\end{aligned}
$$

where $\alpha$ denotes the ratio of females to males in the population, and $m$ denotes the expected share of paternity gained by the second male to mate with a female $(m>$ 0.5 , thus, indicates that the last male to mate with a female has an advantage, and $m<0.5$ that the first male to mate with her has an advantage). The first integral in the above equation represents the fitness gain from mating with previously unmated females, who stand to gain too little to go on to remate. The second integral represents the fitness gain from mating with previously unmated females who will go on to remate. Finally, the third integral represents the fitness gain from mating with previously mated females (for whom the benefits of remating were large enough to outweigh the anticipated costs of acquiring additional toxin).

At a phenotypically stable equilibrium, the level of toxin transfer adopted by males, denoted $t^{*}$, must be that which maximizes an individual male's fitness (given that it is adopted by all other males in the population). This requirement yields the necessary (though not sufficient) condition

$$
\frac{\partial w(t, \hat{t})}{\partial t}=0 \text { for } t=\hat{t}=t^{*}
$$

Differentiating (2), we obtain the following expression for $\partial w(t, \hat{t}) / \partial t$,

$$
\begin{aligned}
\frac{\partial w(t, \hat{t})}{\partial t}= & \alpha m p[c(t+\hat{t})-c(t)][1-c(t)]\left[c^{\prime}(t+\hat{t})-c^{\prime}(t)\right] \\
& +\alpha P[c(t+\hat{t})-c(t)]\left[-c^{\prime}(t)+(1-m) c^{\prime}(t+\hat{t})\right] \\
& +\alpha m P[c(2 \hat{t})-c(\hat{t})] c^{\prime}(t+\hat{t}) \\
& -\alpha c^{\prime}(t+\hat{t}) .
\end{aligned}
$$

Substituting (4) into (3) then yields, after some rearrangement, the following equilibrium condition

$$
\begin{aligned}
P\left(g\left(t^{*}\right)\right)+ & m p\left(g\left(t^{*}\right)\right)\left[1-c\left(t^{*}\right)\right] \\
& -\frac{c^{\prime}\left(2 t^{*}\right)}{c^{\prime}\left(2 t^{*}\right)-c^{\prime}\left(t^{*}\right)}=0,
\end{aligned}
$$

where

$$
g\left(t^{*}\right)=c\left(2 t^{*}\right)-c\left(t^{*}\right) .
$$

To calculate explicit solutions to (5a) and (5b), one must specify the distribution of remating benefit $p(f)$ and the cost function $c(t)$. Having done so, these candidate equilibria must be tested for several further stability properties. First, one must demonstrate that they represent fitness maxima with respect to $t$. Locally, this implies that

$$
\frac{\partial^{2} w(t, \hat{t})}{\partial t^{2}}<0 \text { for } t=\hat{t}=t^{*} .
$$

To ensure that the candidate equilibrium is not just a local but a global fitness maximum, one must additionally demonstrate that

$$
w\left(t, t^{*}\right)<w\left(t^{*}, t^{*}\right) \text { for all } t \neq t^{*} .
$$

Candidate equilibria that satisfy (7) represent "unbeatable" strategies since a mutant individual who transfers less or more toxin than the equilibrium amount $t^{*}$ will suffer reduced fitness compared to other members of the population. Finally, one must determine whether or not these equilibria are continuously stable (see Eshel 1983; Christiansen 1991; Motro 1994; Taylor 1996), that is, whether selection will tend to drive population strategies in the neighborhood of $t^{*}$ toward the equilibrium value. For stability in this sense, it is sufficient that

$$
\frac{\partial}{\partial \hat{t}}\left[\left.\frac{\partial w(t, \hat{t})}{\partial t}\right|_{t=\hat{t}}\right]<0 \text { for } \hat{t}=t^{*}
$$

Only unbeatable strategies that are also continuously stable 
represent likely "endpoints" for the trajectory of an evolving population.

\section{Results}

As stated above, to solve equations (5a) and (5b) explicitly for $t^{*}$, one must specify the distribution of remating benefit $p(f)$ and the cost function $c(t)$. Unfortunately, empirical data on the cumulative impact of seminal toxins on female fitness and on the distribution of remating benefits are lacking. In this section, we therefore present results obtained for the illustrative case in which $f$ is normally distributed (with mean $\mu$ and standard deviation $\sigma$ ), and cost is a power function of the quantity of toxin transferred (i.e., $c(t)=t^{a}$, where greater values of $a$ imply more strongly accelerating costs). The results were calculated numerically since these functions do not yield a simple, analytical solution.

\section{General Features of the Results}

Over the range of parameter values considered, there never exists more than one continuously stable equilibrium at which $t^{*}>0$ (i.e., at which males transfer some toxins during mating). In other words, for each set of parameter values, we can calculate a unique stable level of toxin transfer. However, for a part of this range, there also exists a second, continuously stable equilibrium at $t^{*}=0$, at which no toxins are transferred. Between these two equilibria, which will be referred to as "toxic" and "nontoxic," respectively, there is a point at which the marginal benefits and costs of increasing toxin transfer exactly balance. This third candidate equilibrium is not, however, continuously stable. Rather, it represents the boundary between the basins of attraction of the toxic and nontoxic equilibria. If the population level of toxin transfer is raised above this threshold point, the marginal benefits to an individual of increasing toxin transfer start to outweigh the marginal costs, suggesting that selection will tend to drive the population level still higher, toward the toxic equilibrium. Conversely, if the level of toxin transfer drops below the threshold, the marginal costs start to outweigh the marginal benefits, suggesting that selection will tend to drive the population level even lower, toward the nontoxic equilibrium (note that this is only an approximate summary of the dynamics of the system-although the marginal benefits of increasing transfer outweigh the marginal costs in a population just above the threshold level, a mutant individual who transfers much less toxin may still be able to invade).

The graphs that follow show both the level of toxin transfer at the toxic equilibrium (as a function of various different parameter values) and also the threshold level of transfer. The latter indicates how readily toxicity can become established in a population. A low threshold implies that even a small deviation from the nontoxic state can trigger a transition toward the toxic equilibrium (in the limit, as the threshold drops to 0 , the nontoxic equilibrium ceases to be continuously stable). A high threshold, by contrast, implies that a more substantial deviation is required and, thus, that toxin transfer is harder to establish (see "Discussion").

\section{Sample Solutions}

Figure $1 A$ shows the stable (solid line) and threshold (dotted line) levels of toxin transfer, as a function of $\mu$ (the mean benefit that females stand to gain from remating), for $\sigma=0.25, a=3$, and $m=0.75$. Note that while negative values of $\mu$ imply that the majority of females do best to refrain from remating even in the absence of toxin transfer, there are always some females for whom the ben-
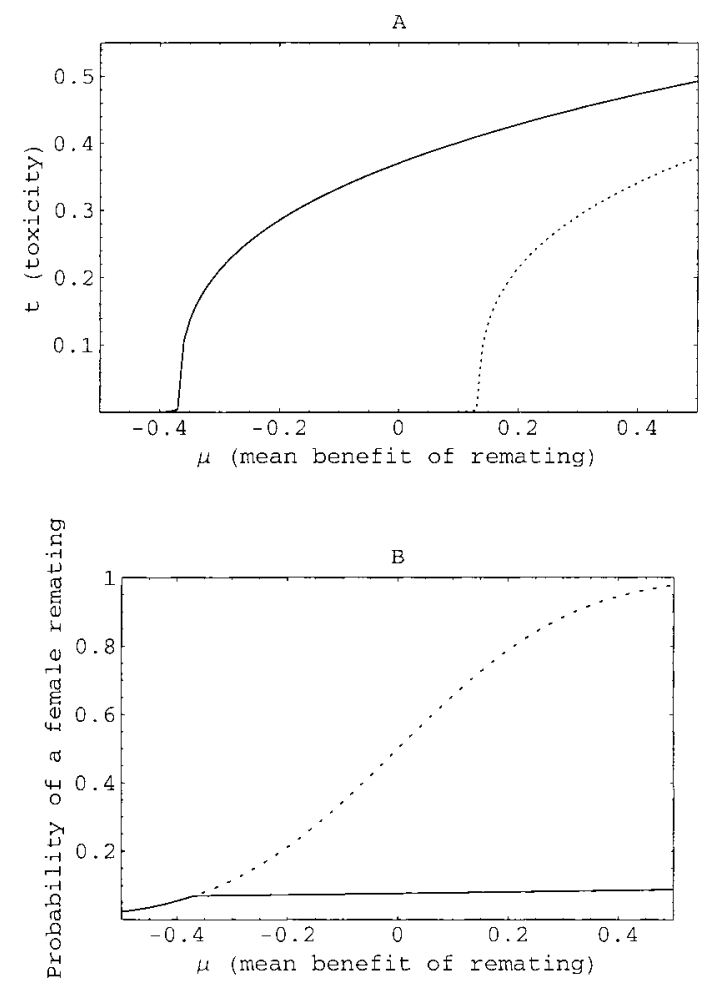

Figure 1: Graph $A$ shows the stable level of toxin transfer at the toxic equilibrium (solid line) and the threshold level required to initiate a transition toward that equilibrium (dotted line), as a function of $\mu$ (the mean benefit that females stand to gain from remating), for $\sigma=0.25$, $a=3$, and $m=0.75$. Graph $B$ shows the probability of a female remating, at the toxic equilibrium (solid line) and at the nontoxic equilibrium (dotted line), over the same parameter range. 
efits of remating outweigh the costs (the lower the value of $\mu$, the rarer are such individuals).

The graph reveals that the outcome of the model is highly sensitive to the expected benefits of remating. The greater the benefits that females stand to gain, the greater the deterrent needed to discourage remating. This leads to an increase in the population level of toxicity required to trigger a transition toward the toxic equilibrium (it is only when there is a sufficiently high population level of toxicity that the toxin transferred by an individual male becomes an adequate deterrent). However, it also leads to greater quantities of toxin being transferred at the toxic equilibrium.

Figure $1 B$ shows the probability that a female will remate, again as a function of $\mu$, for $\sigma=0.25, a=3$, and $m=0.75$, at the toxic equilibrium (solid line) and at the nontoxic equilibrium (dotted line). Unsurprisingly, when no toxins are transferred, the chances of a female remating increase with the mean benefit to be gained by doing so. At the toxic equilibrium, by contrast, the probability of remating remains uniformly low even for high mean benefits. As $\mu$ increases, the greater quantities of toxin transferred by males almost entirely counteract the greater benefits that females stand to gain so that the remating probability shows only a very slight increase.

Figure $2 A$ shows, once again, the stable (solid line) and threshold (dotted line) levels of toxin transfer, this time as a function of $\sigma$ (the standard deviation in remating benefit), for $\mu=0, a=3$, and $m=0.75$. Over a broad range of values, variance in mating benefit has little influence on either the stable or threshold levels of toxicity. As $\sigma$ approaches 0 , however, the equilibrium level of toxin transfer also drops rapidly to 0 . Equally, above a critical value of $\sigma$ (of $\sim 0.35$ in this case), the threshold level of toxicity shows a rapid increase, until the point is reached (at about 0.4 in this case) at which a toxic equilibrium ceases to exist. This reflects the fact that when remating benefit is broadly distributed (i.e., $p(f)$ has high variance), a slight increase in the amount of toxin that a male transfers has very little impact on the proportion of his partners that will go on to remate. Conversely, when remating benefit is tightly clustered around the mean value (i.e., $p(f)$ has low variance), even a small increase in toxicity can induce a large number of females to change their behavior.

As shown in figure $2 B$, the probability of a female remating at the toxic equilibrium increases markedly with the variance in remating benefit. Once again, this reflects the fact that when variance is high, a given increase in toxicity has less influence on the proportion of females that remate (whereas when variance is low, the transfer of even small quantities of toxin is sufficient to render remating unprofitable for the great majority of females).

Having examined the effects of $p(f)$, the distribution
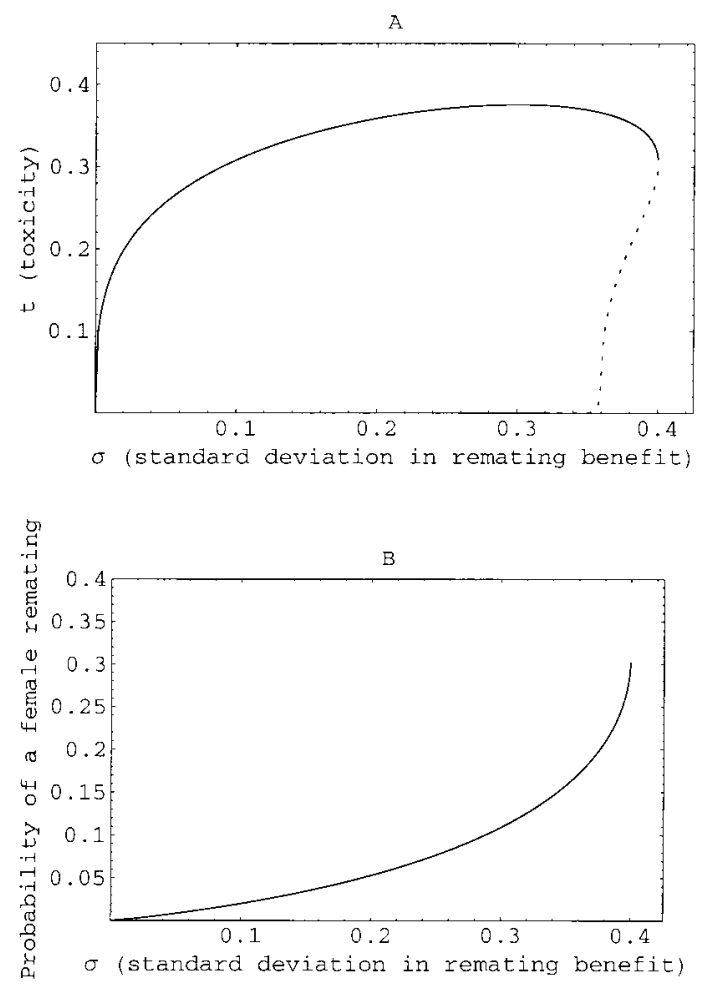

Figure 2: Graph $A$ shows the stable level of toxin transfer at the toxic equilibrium (solid line) and the threshold level required to initiate a transition toward that equilibrium (dotted line) as a function of $\sigma$ (the standard deviation in remating benefit among females), for $\mu=0$ (implying that, in the absence of toxin transfer, $50 \%$ of females will remate), $a=3$, and $m=0.75$. Graph $B$ shows the probability of a female remating, at the toxic equilibrium, over the same parameter range.

of remating benefit among females, we now turn to the cost function $c(t)$. Figure $3 A$ shows the stable (solid line) and threshold (dotted line) levels of toxin transfer as a function of $a$, for $\mu=0, \sigma=0.25$, and $m=0.75$ (higher levels of $a$ imply that cost is a more steeply accelerating function of the quantity of toxin acquired). The graph reveals that a toxic equilibrium is only possible if $a$ exceeds a critical threshold (in this case 2.25 ). Above this point, higher values of $a$ lead to an increase in the quantity of toxin transferred at the toxic equilibrium and a very rapid drop in the threshold level required to initiate a transition toward that equilibrium. For values of $a$ greater than about 2.26, the threshold drops to 0 , implying that the nontoxic equilibrium has ceased to be continuously stable and that any degree of toxicity, however slight, is sufficient to initiate a transition toward the toxic equilibrium. In other words, the more strongly accelerating the costs of the toxin, the easier it is for toxicity to become established in a population and (to a lesser extent) the greater the quantities of toxin that males are likely to employ. As shown 


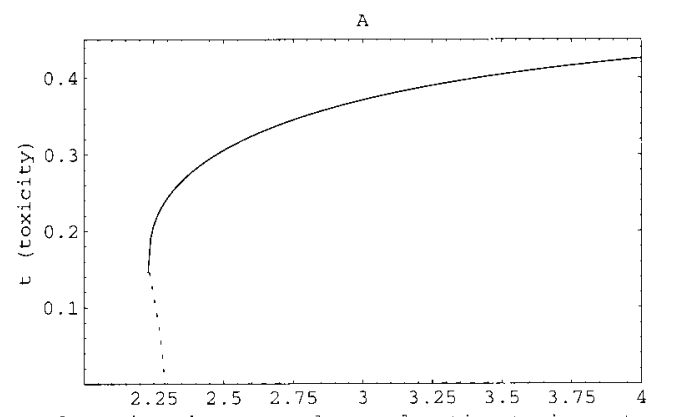

a (determines how strongly accelerating toxin costs are

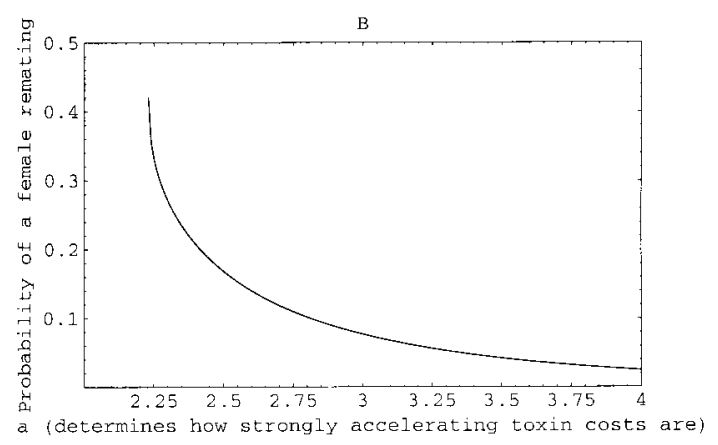

Figure 3: Graph $A$ shows the stable level of toxin transfer at the toxic equilibrium (solid line) and the threshold level required to initiate a transition toward that equilibrium (dotted line), as a function of $a$ (higher values of which imply that cost is a more steeply accelerating function of the quantity of toxin acquired), for $\mu=0$ (implying that, in the absence of toxin transfer, $50 \%$ of females will remate), $\sigma=0.25$, and $m=0.75$. Graph $B$ shows the probability of a female remating, at the toxic equilibrium, over the same parameter range.

in figure $3 B$, the probability of a female remating (at the toxic equilibrium) also decreases markedly as costs become more strongly accelerating.

Finally, we can consider the effects of $m$, the expected share of paternity gained by the last male to mate with a female. Figure $4 A$ shows the stable (solid line) and threshold (dotted line) levels of toxin transfer, as a function of $m$, for $\mu=0, \sigma=0.25$, and $a=3$. The effects of this parameter are similar to those described above for $a$. A toxic equilibrium is only possible if $m$ exceeds a threshold level (of $\sim 0.48$ in this case). Above this, increasing $m$ leads to an increase in the quantity of toxin transferred at the toxic equilibrium, and to a rapid drop in the threshold level required to initiate a transition away from the nontoxic state. In other words, a strong last-male mating advantage both facilitates the initial evolution of toxicity and favors increased levels of toxin transfer. Figure $4 B$ reveals that it also leads to a decrease in the probability of remating at the toxic equilibrium.

Figure 5 gives an overview of these patterns, showing how the equilibrium level of toxin transfer and the thresh- old level required to trigger a transition to the toxic equilibrium vary in relation to $\mu$ (mean remating benefit) for different values of $m$ (last male paternity) and $\sigma$ (standard deviation in remating benefit). It serves to reemphasize that the mean benefit of remating to females is the major determinant of equilibrium toxicity, with high benefits leading to the transfer of larger quantities of toxin. By contrast, last-male mating advantage and variance in remating benefits have a significant influence on the stability and accessibility of a toxic equilibrium. When last-male mating advantage is low and/or there is much variation in remating benefit, a stable toxic equilibrium is less probable and harder to attain (and this is particularly true when the mean remating benefit is large). Provided that a stable toxic equilibrium exists, higher values of $m$ and $\sigma$ also lead to greater levels of toxicity, but these effects are much less marked.
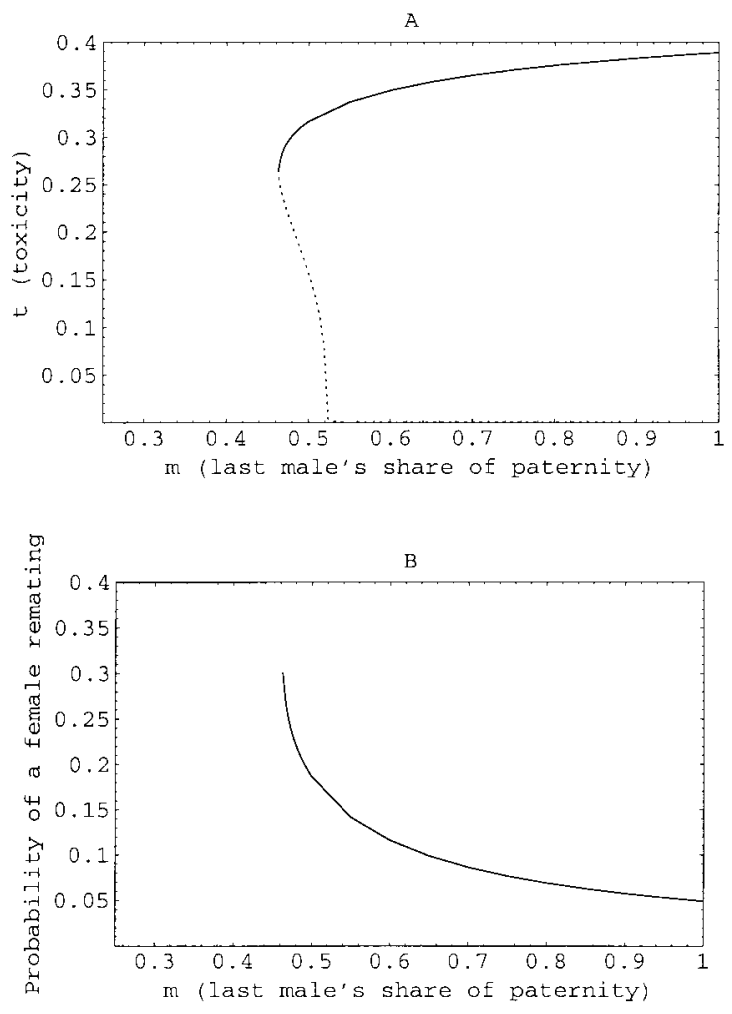

Figure 4: Graph $A$ shows the stable level of toxin transfer at the toxic equilibrium (solid line) and the threshold level required to initiate a transition toward that equilibrium (dotted line), as a function of $m$ (the expected share of paternity gained by the last male to mate with a female), for $\mu=0$ (implying that, in the absence of toxin transfer, $50 \%$ of females will remate), $\sigma=0.25$, and $a=3$. Graph $B$ shows the probability of a female remating, at the toxic equilibrium, over the same parameter range. 


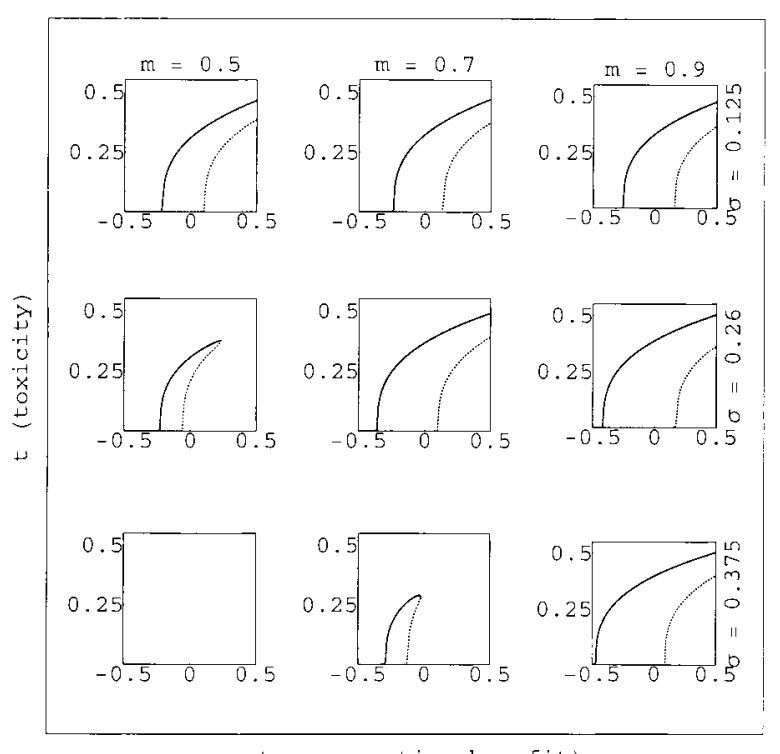

$\mu$ (mean remating benefit)

Figure 5: The stable level of toxin transfer at the toxic equilibrium (solid line) and the threshold level required to initiate a transition toward that equilibrium (dotted line), as a function of $\mu$ (the mean benefit that females stand to gain from remating), for different values of $m$ ( 0.5 for the first column of graphs, 0.7 for the second, and 0.9 for the third) and $\sigma(0.125$ for the first row of graphs, 0.26 for the second, and 0.375 for the third).

\section{Discussion}

These results indicate that seminal toxins can evolve as a result of conflict between males and females over remating. Provided that the fitness cost of the toxin increases with the amount acquired, and does so at an accelerating rate, a female who receives a large dose from her first mate is less likely to benefit from remating. Under these circumstances, as the model shows, selection can favor males who transfer larger quantities of the toxin because they enjoy a reduced risk of sperm competition. Moreover, this argument applies to other damaging mating tactics as well, not only to toxin transfer. Whenever cumulative damage has an accelerating impact on female fitness, selection can favor males that inflict greater harm on their mates. Sexual conflict over remating can thus potentially explain the evolution of morphological and behavioral traits ranging from genital spines and barbs to sexual aggression (see also Gowaty and Buschhaus 1998).

Toxin transfer and other harmful mating tactics should be more common (and involve greater levels of damage) in species with greater last-male mating advantage, since the increased threat from sperm competition in these cases magnifies the benefits that a male stands to gain by deterring remating. Even if such tactics are evolutionarily stable, however, their initial origin may prove problematic.
When none of the other males in a population incorporate harmful products in their semen, for instance, an individual mutant who does so gains no advantage (since it is only the risk of acquiring a double dose that deters females from remating). Instead, he only suffers the cost of a reduction in his mate's reproductive success. The nontoxic state, in other words, may also be evolutionarily stable, which raises the question of how a population can make the initial transition from a nontoxic to a toxic equilibrium.

One possibility is that sperm competition may be responsible for the initial evolution of a certain degree of seminal toxicity; in line with this suggestion, there is evidence in Drosophila melanogaster that toxic accessory gland products increase a male's fertilization success, possibly by disabling or destroying the sperm of previous mates (see Harshman and Prout 1994 and Chapman et al. 1995). Once toxin transfer becomes established in this way, the possibility of restraining females from remating will start to play a role, giving rise to selection for further increases in toxicity. Sperm competition could thus trigger the transition from a nontoxic state to a toxic equilibrium of the kind examined in the model. Other forms of mating damage, too, might arise as a consequence of intrasexual competition or other selective pressures, only subsequently to become a means of manipulating female remating behavior. Aggressive forced copulation, for instance, may evolve as a means to gain extra mating opportunities (see Gowaty and Buschaus 1998), but such aggression could later also be maintained or further elaborated because of its impact on female remating.

Intriguingly, though, it may not always be necessary to invoke an alternative explanation for the initial origin of harmful mating tactics. Under some circumstances (e.g., with strong last-male mating advantage and/or strongly accelerating costs of a toxin), this model reveals that the threshold level of toxin transfer required to trigger a transition to the toxic evolutionarily stable strategy (ESS) drops to 0 . Under these circumstances, even an arbitrarily small perturbation of the nontoxic equilibrium is enough to initiate evolution toward the alternative, toxic equilibrium. This reflects the assumption that as the amount of toxin a female acquires tends to 0 , so too does the marginal cost of increasing quantities of the harmful substance. Whenever the marginal costs of accumulated damage tend to 0 in this way, harmful mating tactics can readily become established in a population, because their initial origin has negligible impact on female fitness.

The model predicts that seminal toxins and other harmful mating tactics should be particularly common in populations and/or species with higher last-male mating advantage. Unfortunately, however, one would expect precisely the same pattern if toxicity were primarily an 
adaptation to sperm competition (from the latter perspective, toxins are a cause, rather than a consequence, of strong last-male mating advantage). Empirical tests of our hypothesis would, therefore, do better to focus on other predictions, for instance, that seminal fluids should have an accelerating dose-dependent effect on female fitness (and on the likelihood of remating) or that accumulated physical damage should impose increasing fitness costs on females that remate. Chapman et al. (1995) have, in fact, shown that in D. melanogaster, the death rates of females mated to mutant males differing in the amount of main cell fluid transferred during copulation $(0 \%, 1 \%, 3 \%$, and $100 \%$ of normal doses) increased with the quantity transferred. However, their experiments do not allow one to determine the quantitative relationship between the amount of seminal fluids transferred and female life span and, thus, determine whether or not the toxin has an accelerating impact.

Turning to the possibilities for future theoretical work, several simplifying assumptions of the model are open to modification. For instance, we have assumed that the costs to males of toxin production (or of any other harmful behavior) are negligible compared to the reduction in female reproductive success it entails. The incorporation of a cost of production into the model would tend to lower the equilibrium level of transfer and to raise the threshold required to initiate a transition toward the toxic ESS. At the same time, however, we have also assumed that the costs that a male inflicts on his mates adversely affect his own reproductive success, too. Costs that take the form of a reduction in longer-term survival will have no impact on the male's reproductive success, though they will, nevertheless, influence female remating behavior. Incorporating such long-term costs to females into the model would thus tend to raise the equilibrium level of toxic transfer or damage and to lower the threshold required to initiate a transition toward the harmful ESS (i.e., to counteract the effects of any cost of toxin production).

Second, we have not allowed for differential male behavior in relation to female mating status. The optimal level of damage or toxicity, from a male's point of view, represents a trade-off between the benefits of deterring remating (which boosts paternity) and the costs of impairing female fitness (which may also reduce the male's reproductive success). Consequently, a male would do best to reduce the harm inflicted on females, who have already mated one or more times, because they are less likely to mate again even if he imposes no such mating costs. Conversely, a virgin female is more likely to remate after copulating with the male in question so that he does best to inflict greater harm. It would be interesting to incorporate the possibility of multiple remating by females and of differential behavior by males into the model. However, it seems likely that early on in the evolution of damaging mating tactics, such complex strategies on the part of males are unlikely (particularly if female mating status is hard to determine).

Finally, we have not considered the possibility of females evolving resistance to damaging male tactics (Rice 1996). The effects of this are hard to predict and would depend on the way in which it affected the shape of the cost curve $c(t)$. Increased resistance might, on the one hand, force males to increase the level of damage inflicted or to produce greater quantities of toxin in order to deter remating; if this is costly, it could render such tactics unstable. On the other hand, if resistance were most effective against small quantities of damage or toxin (thus, effectively rendering the cost curve more steeply accelerating), it might actually favor the evolution of damaging mating tactics, by reducing the deleterious effects imposed by the first male, while leaving the cumulative damage imposed by the second mating largely unchanged.

\section{Acknowledgments}

We thank T. Chapman, E. Fjerdingstad, and L. Partridge for helpful comments and the Swiss National Science Foundation for financial support.

\section{Literature Cited}

Baur, B. 1998. Sperm competition in molluscs. Pages 255-305 in T. R. Birkhead and A. P. Møller, eds. Sperm competition and sexual selection. Academic Press, London.

Birkhead, T. R. 1998. Sperm competition in birds: mechanisms and function. Pages 579-622 in T. R. Birkhead and A. P. Møller, eds. Sperm competition and sexual selection. Academic Press, London.

Bryan, W. R., and M. B. Shimkin. 1943. Quantitative analysis of dose-response data obtained with three carcinogenic hydrocarbons in strain $\mathrm{C} 3 \mathrm{H}$ male mice. Journal of the National Cancer Institute 3:503-531.

Chapman, T. 1992. A cost of mating with males that do not transfer sperm in female Drosophila melanogaster. Journal of Insect Physiology 38:223-227.

Chapman, T., S. Trevitt, and L. Partridge. 1994. Remating and male-derived nutrients in Drosophila melanogaster. Journal of Evolutionary Biology 7:51-69.

Chapman, T., L. F. Liddle, J. M. Kalb, M. F. Wolfner, and L. Partridge. 1995. Cost of mating in Drosophila melanogaster females is mediated by male accessory gland products. Nature (London) 373:241-244.

Chapman, T., T. Miyatake, H. K. Smith, and L. Partridge. 1998. Interactions of mating, egg production and death rates in females of the Mediterranean fruit fly, Ceratitis 
capitata. Proceedings of the Royal Society of London B, Biological Sciences 265:1879-1894.

Chen, P. S. 1984. The functional morphology and biochemistry of insect male accessory glands and their secretions. Annual Review of Entomology 29:233-255.

Christiansen, F. B. 1991. On conditions for evolutionary stability for a continuously varying character. American Naturalist 138:37-50.

Cordero, C. 1995. Ejaculate substances that affect female insect reproductive physiology and behavior: honest or arbitrary traits? Journal of Theoretical Biology 174: 453-461.

Daly, M. 1978. The cost of mating. American Naturalist 112:771-774.

Eberhard, W. G. 1985. Sexual selection and animal genitalia. Harvard University Press, London.

Eshel, I. 1983. Evolutionary and continuous stability. Journal of Theoretical Biology 103:99-111.

Fowler, K., and L. Partridge. 1989. A cost of mating in female fruitflies. Nature (London) 338:760-761.

Gomendio, M., A. H. Harcourt, and E. R. S. Roldán. 1998. Sperm competition in mammals. Pages 667-755 in $\mathrm{T}$. R. Birkhead and A. P. Møller, eds. Sperm competition and sexual selection. Academic Press, London.

Gowaty, P. A., and N. Buschhaus. 1998. Ultimate causation of aggressive and forced copulation in birds: female resistance, the CODE hypothesis, and social monogamy. American Zoologist 38:207-225.

Gromko, M. H., M. E. A. Newport, and M. G. Kortier. 1984. Sperm dependence of female receptivity in Drosophila melanogaster. Evolution 38:1273-1282.

Happ, G. H., M. E. Schroder, and J. C. H. Wang. 1970. Effects of male and female scent on reproductive maturation in young female Tenebrio molitor. Journal of Insect Physiology 16:1543-1548.

Harshman, L. G., and T. Prout. 1994. Sperm displacement without sperm transfer in Drosophila melanogaster. Evolution 48:758-766.

Harshman, L. G., A. A. Hoffman, and T. Prout. 1988. Environmental effects on remating in Drosophila melanogaster. Evolution 42:312-321.

Jennions, M. D., and M. Petrie. 2000. Why do females mate multiply? a review of the genetic benefits. Biological Reviews of the Cambridge Philosophical Society 75: 21-64.

Keller, L., and H. K. Reeve. 1995. Why do females mate with multiple males? the sexually selected sperm hypothesis. Advances in the Study of Behavior 24:291-315.

LeBoeuf, B. J., and S. L. Mesnick. 1991a. Sexual behavior of male northern elephant seals. I. Lethal injuries to adult females. Behaviour 116:142-162.

- $1991 b$. Sexual behavior of male northern elephant seals. II. Female response to potentially injurious encounters. Behaviour 117:262-280.

Lewis, S. M., and S. N. Austad. 1994. Sexual selection in flour beetles: the relationship between sperm precedence and male olfactory attractiveness. Behavioral Ecology 5: 219-224.

Madsen, T., R. Shine, J. Loman, and T. Hakansson. 1992. Why do female adders copulate so frequently? Nature (London) 355:440-441.

Motro, U. 1994 Evolutionary and continuous stability in asymmetric games with continuous strategy sets- the parental investment conflict as an example. American Naturalist 144:229-241.

Murphy, S. D., and K. L. Cheever. 1968. Effects of feeding insecticides: inhibition of carboxylesterase and cholinesterase activities in rats. Archives of Environmental Health 17:749-756.

Olsson, M., and T. Madsen. 1998. Sexual selection and sperm competition in reptiles. Pages 503-577 in T. R. Birkhead and A. P. Møller, eds. Sperm competition and sexual selection. Academic Press, London.

Parker, G. A. 1970. Sperm competition and its evolutionary consequences in the insects. Biological Reviews of the Cambridge Philosophical Society 45:525-567.

- 1984. Sperm competition and the evolution of animal mating strategies. Pages 2-60 in R. I. Smith, ed. Sperm competition and the evolution of animal mating systems. Academic Press, London.

Radwan, J., and M. Rysinska. 1999. Effect of mating frequency on female fitness in Caloglyphus berlesei (Astigmata: Acaridae). Experimental and Applied Acarology 25:399-409.

Rice, W. R. 1996. Sexually antagonistic male adaptation triggered by experimental arrest of female evolution. Nature (London) 381:232-234.

Ridley, M. 1988. Mating frequency and fecundity in insects. Biological Reviews of the Cambridge Philosophical Society 63:509-549.

- 1993. Clutch size and mating frequency in parasitic hymenoptera. American Naturalist 142:893-910.

Roger, J. M., and R. J. Kavlock. 1996. Developmental toxicity. Pages 301-322 in C. D. Klaassen, ed. Toxicology: the basic science of poisons. McGraw-Hill, New York.

Shuey, D. L., C. Lau, T. R. Lodgson, R. M. Zucker, K. H. Elstein, M. G. Narotsky, R. W. Setzer, R. J. Kavlock, and J. M. Rogers. 1994. Biologically based dose-response modelling in developmental toxicology: biochemical and cellular sequelae of 5-fluorouracil exposure in the developing rat. Toxicology and Applied Pharmacology 126:129-144.

Simmons, L. W., and M. T. Siva-Jothy. 1998. Sperm competition in insects: mechanisms and the potential for selection. Pages 341-434 in T. R. Birkhead and A. P. 
Møller, eds. Sperm competition and sexual selection. Academic Press, London.

Taylor, P. D. 1996. Inclusive fitness arguments in genetic models of behavior. Journal of Mathematical Biology 34:654-674.

Thornhill, R., and J. Alcock. 1983. The evolution of insect mating systems. Harvard University Press, Cambridge, Mass.

von Helversen, D., and O. von Helversen. 1991. Pre-mating sperm removal in the bushcricket Metaplastes or- natus Ramme 1931 (Orthoptera, Tettigonoidea, Phaneropteridae). Behavioral Ecology and Sociobiology 28: 391-396.

Walker, W. F. 1980. Sperm utilization strategies in nonsocial insects. American Naturalist 115:780-799.

Yasui, Y. 1998. The "genetic benefits" of female multiple mating reconsidered. Trends in Ecology \& Evolution 13: 246-250.

Associate Editor: Marlene Zuk 